Author affiliations and support information (if applicable) appear at the end of this article.

Published at jco.org on January 10, 2018.

Processed as a Rapid Communication manuscript.

Clinical trial information: NCT02091141

Corresponding author: John D. Hainsworth, MD, Sarah Cannon Research Institute, 1100 Charlotte Ave, Suite 800, Nashville, TN 37203; e-mail: john. hainsworth@scresearch.net.

() 2018 by American Society of Clinical Oncology

0732-183X/18/3606w-536w/\$20.00

\title{
Targeted Therapy for Advanced Solid Tumors on the Basis of Molecular Profiles: Results From MyPathway, an Open-Label, Phase IIa Multiple Basket Study
}

John D. Hainsworth, Funda Meric-Bernstam, Charles Swanton, Herbert Hurwitz, David R. Spigel, Christopher Sweeney, Howard Burris, Ron Bose, Bongin Yoo, Alisha Stein, Mary Beattie, and Razelle Kurzrock

\section{$\begin{array}{llllllll}A & B & S & T & R & A & C & T\end{array}$}

\section{Purpose}

Detection of specific molecular alterations in tumors guides the selection of effective targeted treatment of patients with several types of cancer. These molecular alterations may occur in other tumor types for which the efficacy of targeted therapy remains unclear. The MyPathway study evaluates the efficacy and safety of selected targeted therapies in tumor types that harbor relevant genetic alterations but are outside of current labeling for these treatments.

\section{Methods}

MyPathway (ClinicalTrials.gov identifier: NCT02091141) is a multicenter, nonrandomized, phase Ila multiple basket study. Patients with advanced refractory solid tumors harboring molecular alterations in human epidermal growth factor receptor-2, epidermal growth factor receptor, v-raf murine sarcoma viral oncogene homolog B1, or the Hedgehog pathway are treated with pertuzumab plus trastuzumab, erlotinib, vemurafenib, or vismodegib, respectively. The primary end point is investigatorassessed objective response rate within each tumor-pathway cohort.

\section{Results}

Between April 1, 2014 and November 1, 2016, 251 patients with 35 different tumor types received study treatment. The efficacy population contains 230 treated patients who were evaluated for response or discontinued treatment before evaluation. Fifty-two patients (23\%) with 14 different tumor types had objective responses (complete, $n=4$; partial, $n=48$ ). Tumor-pathway cohorts with notable objective response rates included human epidermal growth factor receptor-2-amplified/ overexpressing colorectal (38\% [14 of 37]; $95 \% \mathrm{Cl}, 23 \%$ to $55 \%$ ) and v-raf murine sarcoma viral oncogene homolog B1 V600-mutated non-small-cell lung cancer (43\% [six of 14]; $95 \% \mathrm{Cl}, 18 \%$ to $71 \%)$.

\section{Conclusion}

The four currently approved targeted therapy regimens in the MyPathway study produced meaningful responses when administered without chemotherapy in several refractory solid tumor types not currently labeled for these agents.

\section{J Clin Oncol 36:536-542. (C) 2018 by American Society of Clinical Oncology}

\section{INTRODUCTION}

Molecular profiling is routinely used to guide the selection of targeted therapies for the treatment of patients with lung, breast, colon, and other types of cancer. When used in molecularly identified patients with indicated tumor types, agents targeting human epidermal growth factor receptor-2 (HER2), ${ }^{1}$ epidermal growth factor receptor (EGFR), ${ }^{2} \mathrm{v}$-raf murine sarcoma viral oncogene homolog B1 (BRAF), ${ }^{3}$ and the Hedgehog pathway ${ }^{4}$ have been among the most effective therapies introduced during the last 20 years.
Molecular alterations in these genes and pathways also occur in a variety of nonindicated tumor types. The low incidence of the targeted molecular alterations in these tumor types (usually $<5 \%$ ) has made it difficult to recruit sufficient numbers of patients into traditional drug development studies, although activity has been documented in anecdotal reports. ${ }^{5-12}$

Basket studies provide the opportunity to evaluate the efficacy of targeted therapies in patient populations defined by the presence of specific molecular tumor abnormalities, rather than by primary site or tumor histology. ${ }^{13,14}$ Results of 
several small basket studies have recently been reported. In one such study (ClinicalTrials.gov identifier: NCT01524978), treatment with vemurafenib produced responses in 24 of 95 patients $(25 \%)$ with $B R A F$ V600-mutated tumor types other than melanoma and colorectal cancer. ${ }^{15}$ Such data suggest that certain molecular alterations are important in determining the response to targeted treatment, independent of tumor type. ${ }^{16}$ However, multiple variables influence the efficacy of targeted therapy, including the biology and relevance of the target, the mechanism of target inhibition, the tumor type, the presence of other molecular alterations, and the therapy selected.

MyPathway is an ongoing, multicenter, phase IIa study that combines multiple basket studies under an adaptable master protocol. The objective of the study is to evaluate the efficacy of treatments targeting molecular alterations in HER2 (pertuzumab plus trastuzumab), BRAF (vemurafenib), Hedgehog pathway (vismodegib), or EGFR (erlotinib) in patients with tumor types outside of current labeling for these treatment regimens. In this report, we summarize the efficacy results in the first 230 treated patients who were efficacy evaluable.

\section{METHODS}

\section{Patients}

Eligible patients are required to have refractory, metastatic solid tumors containing targetable, pathway-activating, molecular alterations in HER2, EGFR, BRAF, or the Hedgehog pathway. Molecular profiling was not conducted as part of this study; rather, eligible patients were required to have previous profiling demonstrating one of the target alterations. Molecular testing by various local Clinical Laboratory Improvement Amendments (CLIA)-approved laboratories was acceptable in this study. Molecular profiling must have been performed on tissue obtained at the most recent tumor biopsy.

Additional eligibility requirements include measurable or evaluable lesions ${ }^{17}$ and an Eastern Cooperative Oncology Group performance status score of 0,1 , or 2 . Patients with tumor types for which the study treatments are approved or in active development are not eligible. Specific tumor mutations with evidence suggesting inactivity of the study drugs are also ineligible. All patients also need to fulfill additional eligibility criteria pertinent to the specific therapeutic agents (Data Supplement).

Molecular alterations eligible for MyPathway as well as required testing methods are detailed in Table 1. Tumors with HER2 amplification, overexpression, and/or activating mutations were eligible. For EGFR and BRAF, known or putative activating mutations were required. Eligible Hedgehog pathway alterations included gain-of-function mutations of Smoothened (SMO) or loss-of-function mutations of Patched Homolog-1 $(\mathrm{PTCH}-1)$. Unusual mutations considered possible activating mutations for any of the pathways (or loss of function mutations for $\mathrm{PTCH}-1$ ) were permitted if previously reported in at least two unique specimens in the Catalogue of Somatic Mutations in Cancer database. ${ }^{6}$

The above protocol-specified molecular alterations were identified through local CLIA-approved laboratory reports and reviewed by the study medical monitor for eligibility. For unusual molecular alterations, the medical monitor consulted appropriate members of the MyPathway Steering Committee regarding eligibility. Scanned laboratory reports are kept in a separate database from clinical trial data.

\section{Trial Design}

MyPathway (ClinicalTrials.gov identifier: NCT02091141) is a multicenter, nonrandomized, open-label, multiple basket phase IIa trial (Fig 1). Eligible patients are assigned to a specific treatment cohort on the basis of the presence of a relevant target molecular alteration in their tumor biopsy. ${ }^{19}$ Patients with two or more study-eligible alterations may be treated for the

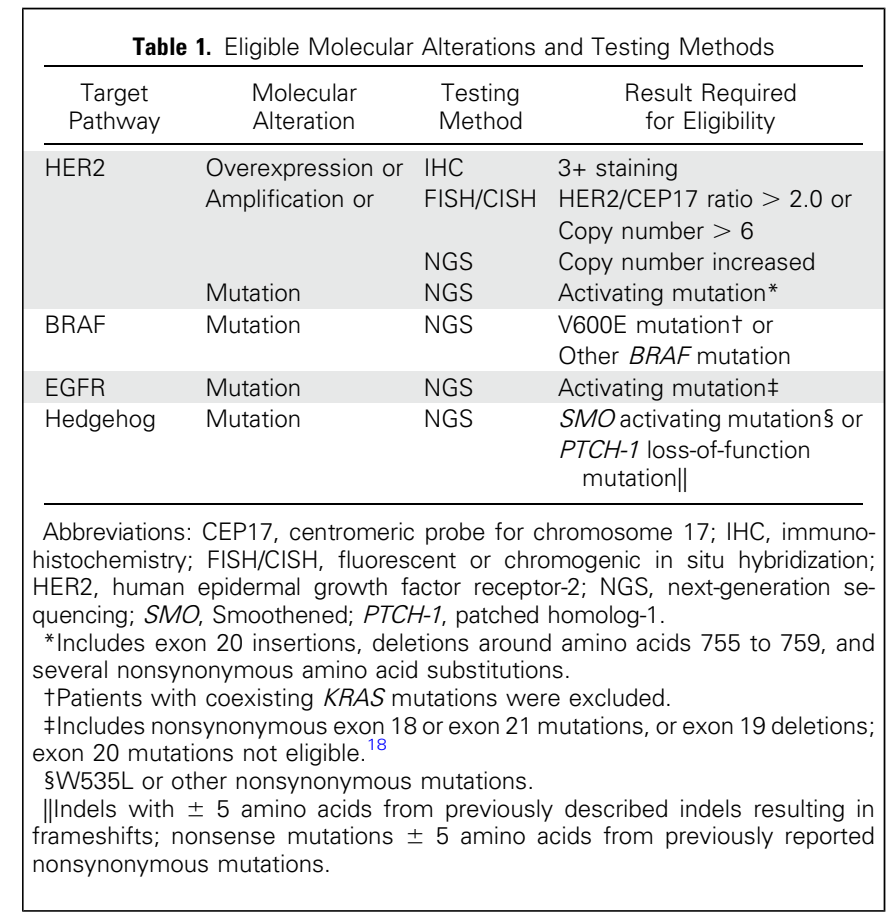

alteration that the investigator considers most clinically relevant and may subsequently enter a different treatment cohort if they remain eligible.

Dosages of study treatments are in accordance with the United States package inserts for each product ${ }^{20-24}$ and are administered without systemic chemotherapy. Patients with HER2 molecular alterations are treated with intravenous pertuzumab ( $840 \mathrm{mg}$ loading dose followed by $420 \mathrm{mg}$ every 3 weeks) plus intravenous trastuzumab $(8 \mathrm{mg} / \mathrm{kg}$ loading dose followed by $6 \mathrm{mg} / \mathrm{kg}$ every 3 weeks). Patients with EGFR-activating mutations are treated with erlotinib (150 mg orally once daily). Patients with $B R A F$ mutations are administered vemurafenib (960 mg orally twice daily), and those with Hedgehog pathway alterations receive vismodegib (150 mg orally once daily). Patients receive treatment of two cycles (6 weeks for pertuzumab plus trastuzumab; 8 weeks for all oral agents) and are then evaluated for response. Patients with objective response or stable disease (SD) continue therapy, with repeat evaluations every two cycles for the first 24 weeks, followed by evaluations every 12 weeks, until tumor progression, unacceptable toxicity, or other discontinuation criteria are met.

\section{Trial Oversight}

The MyPathway protocol was approved by the institutional review board/ethics committee at each trial center. Written informed consent is obtained before any screening procedures. Genentech and the Sarah Cannon Research Institute designed the trial in collaboration with the MyPathway Steering Committee. The MyPathway Steering Committee meets three times per year, and when necessary, to review safety and efficacy data and preplanned futility analyses. All authors have access to the data and vouch for the accuracy and completeness of the analyses reported and for the fidelity of the trial to the protocol.

\section{Study End Points}

The primary efficacy end point for MyPathway is the objective response rate (ORR). Responses are assessed by the investigator according to Response Evaluation Criteria in Solid Tumors (RECIST) version 1.1. ${ }^{17}$ Confirmation of response (per RECIST) is not required. Secondary efficacy end points include duration of response (DOR), progression-free survival, and 1-year overall survival. DOR is measured from the date of first documentation of an objective response to the date of tumor progression/death or 


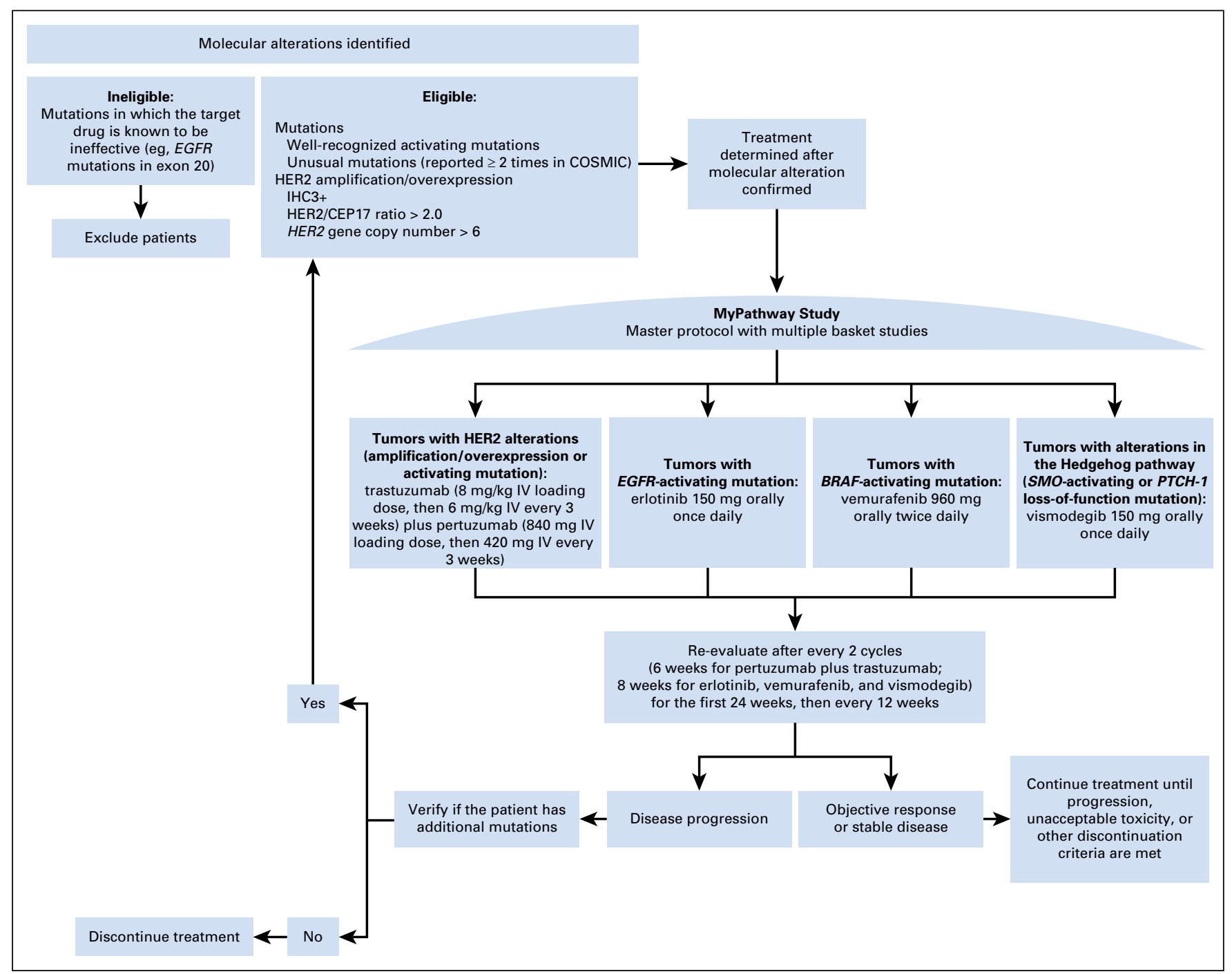

Fig 1. MyPathway study design. BRAF, murine sarcoma viral (v-raf) oncogene homolog B1; CEP17, centromeric probe for chromosome 17; COSMIC, Catalogue of Somatic Mutations in Cancer; EGFR, epidermal growth factor receptor; HER2, human epidermal growth factor receptor-2; IHC, immunohistochemistry; IV, intravenous; PTCH-1, patched homolog-1; q3w, every 3 weeks; SMO, smoothened.

the last tumor assessment (if there was no progressive disease or death). Efficacy end points are evaluated separately for each tumor-pathway cohort, as defined by specific tumor type and molecular alteration.

\section{Statistical Analysis}

Analyses of ORR were performed in the efficacy analysis population, which included patients with measurable disease who were treated and had been evaluated for tumor response or who had been discontinued from treatment for any reason before the first response evaluation. Analyses of the DOR include the efficacy analysis patients who had objective responses. The data cutoff date for this interim efficacy analysis population was November 1, 2016.

ORRs were calculated for each tumor-pathway cohort. The 95\% CIs of ORR were constructed using exact binomial distribution (ClopperPearson estimation method). The Kaplan-Meier approach was used to estimate median DORs and their 95\% CIs and median follow-up duration.

For each tumor-pathway cohort, Simon's two-stage design was used to make a preliminary assessment of treatment activity. For treatmentresistant tumor types (eg, non-small-cell lung cancer [NSCLC] and biliary), at least one response in the first 12 patients was required for cohort expansion; otherwise, the cohort was closed for futility (testing hypothesis:
ORR, $5 \%>20 \%$ at $10 \%$ one-sided type I error, $80 \%$ power). The MyPathway Steering Committee was consulted to determine treatmentresistant tumor types. For less-resistant tumor types (eg, colorectal, ovary), at least two responses in the first 13 patients were required for cohort expansion (testing hypothesis: ORR, 10\% $v 25 \%$ at $10 \%$ one-sided type I error, $80 \%$ power). If preliminary activity was demonstrated, accrual to the cohort continued to a maximum of 75 patients.

The initial study protocol did not include formal plans for separate analyses of cohorts with HER2 amplification/overexpression and HER2 mutation. However, apparent differences in tumor types and accrual of adequate numbers for analysis in each group led us to consider them separately. Similar considerations led to the separate analyses of groups with BRAF V600E mutations versus other BRAF mutations.

\section{RESULTS}

\section{Patients}

From April 1, 2014 to November 1, 2016, 251 patients with 35 different tumor types were enrolled from 38 centers in the United 
States. The efficacy analysis population includes 230 patients; the remaining 21 patients had not yet reached the first efficacy evaluation. Patient demographics for the efficacy analysis group included: median age 62 years (range, 23 to 86 years), $51.0 \%$ male, $91.6 \%$ good performance status (Eastern Cooperative Oncology Group 0 or 1), and a median of 2.5 prior systemic treatment regimens (range, 0 to 9). In 107 patients, molecular alterations were detected in tumor tissue obtained at the time of diagnosis (primary site, $\mathrm{n}=66$; metastatic site, $\mathrm{n}=37$; unknown site, $\mathrm{n}=4)$, whereas 123 patients had alterations detected in subsequent biopsies. Table 2 lists the tumor types and molecular alterations.

Tumor molecular alterations identified in the 230 patients were as follows: HER2, 151 (66\%); BRAF, 49 (21\%); Hedgehog, 21 (9\%); and EGFR, nine (4\%). Of the 151 patients enrolled in the HER2 arm, 114 exhibited HER2 amplification/overexpression (12 of whom also had HER2 mutations), 36 had HER2-activating mutations (without amplification detected by next-generation sequencing), and one had an RBMS-NRG1 fusion (an alteration suggested to result in HER2 pathway activation). Twenty-six of the 49 patients with BRAF-mutated tumors had V600E mutations, whereas 23 patients had tumors with a variety of other $B R A F-$ activating mutations.

\section{Efficacy}

The median duration of follow-up for the efficacy population was 9.7 months (range, 0.3 to 22.1 months). Fifty-two patients (23\%) with 14 tumor types had objective responses (four complete responses [CR], 48 partial responses [PR]), and 26 additional patients had SD for $>120$ days. Responses were seen with each of the four targeted treatments.

Four tumor-pathway cohorts have enrolled at least 12 patients and had protocol-mandated efficacy review: HER2 amplified/ overexpressing colorectal cancer $(\mathrm{n}=37)$, HER2 amplified/ overexpressing NSCLC $(\mathrm{n}=16)$, HER2-mutated NSCLC $(\mathrm{n}=14)$,

\begin{tabular}{|c|c|c|c|c|c|}
\hline Primary Site & HER2 & BRAF & $\begin{array}{l}\text { Hedgehog } \\
\text { Pathway }\end{array}$ & EGFR & Total \\
\hline Lung, non-small-cell & 30 & 21 & 3 & 0 & 54 \\
\hline Colorectal & 40 & 2 & 0 & 0 & 42 \\
\hline Biliary & $11^{*}$ & 3 & 0 & 1 & 15 \\
\hline Ovary & 8 & 4 & 2 & 0 & 14 \\
\hline Bladder & 13 & 0 & 0 & 0 & 13 \\
\hline Pancreas & 9 & 4 & 0 & 0 & 13 \\
\hline Uterus & 7 & 0 & 0 & 0 & 7 \\
\hline Breast & $2 \dagger$ & 0 & 2 & 2 & 6 \\
\hline Salivary gland & 5 & 0 & 1 & 0 & 6 \\
\hline Small intestine & 4 & 0 & 1 & 1 & 6 \\
\hline Prostate & 1 & 3 & 1 & 0 & 5 \\
\hline Unknown primary & 1 & 3 & 1 & 0 & 5 \\
\hline Other (21 tumor types) & 20 & 9 & 10 & 5 & 44 \\
\hline Total & $151(66 \%)$ & $49(21 \%)$ & $21(9 \%)$ & $9(4 \%)$ & 230 \\
\hline \multicolumn{6}{|c|}{$\begin{array}{l}\text { NOTE. } N=230 \text {. } \\
\text { Abbreviations: BRAF, murine sarcoma viral (v-raf) oncogene homolog B1; } \\
\text { EGFR, epidermal growth factor receptor; HER2, human epidermal growth factor } \\
\text { receptor-2. } \\
\text { *One patient had a tumor with an RBMS-NRG1 fusion. } \\
\text { †Both had HER2 mutations without amplification or overexpression. }\end{array}$} \\
\hline
\end{tabular}

and BRAF V600E-mutated NSCLC $(\mathrm{n}=14)$. All four cohorts exceeded minimum efficacy criteria, and accrual has been extended. Several other cohorts have shown evidence of activity but have not reached the size for formal review. No tumorpathway cohorts have been closed for lack of efficacy. However, the study was closed to further accrual of BRAF non-V600 mutations because of the low response rate (one of $23 ; 4 \%$ ) in diverse tumor types.

\section{Patients With HER2 Amplification/Overexpression}

Thirty of 114 patients ( $26 \%$; 95\% CI, 19\% to 35\%) with HER2 amplification/overexpression had objective responses to treatment with trastuzumab plus pertuzumab (two CR, $28 \mathrm{PR}$ ). Objective responses were seen in nine primary tumor types: colorectal, bladder, biliary, salivary gland, NSCLC, pancreas, ovary, prostate, and skin (apocrine; Table 3).

Patients with HER2-amplified/overexpressing metastatic colorectal cancer composed the largest tumor-pathway cohort. In this group of 37 patients with refractory disease (median, four previous lines of therapy), treatment with trastuzumab plus pertuzumab produced PRs in 14 patients (38\%; 95\% CI, 23\% to 55\%; Fig 2A). An additional four patients had SD $>120$ days. The median DOR was 11 months (range, $<1$ to $16+$ months; $95 \% \mathrm{CI}, 2.8$ months to not estimable).

Although the other tumor-pathway cohorts are relatively small, treatment with trastuzumab plus pertuzumab showed substantial activity in several other refractory tumor types. Three of nine patients (33\%; 95\% CI, $8 \%$ to $70 \%$ ) with advanced bladder cancer had responses (one CR ongoing at 15 months; two PR lasting 1 and 6 months), and two patients had SD $>120$ days (Fig 2B). Two of seven patients (29\%; 95\% CI, $4 \%$ to $71 \%$ ) with biliary cancer had PR, and three had SD $>120$ days (Fig 2C). Finally, four of five patients with salivary gland carcinoma $(80 \%$; $95 \%$ CI, $28 \%$ to $>99 \%$ ) had responses (all PR).

\begin{tabular}{|c|c|c|c|c|c|}
\hline \multirow[b]{2}{*}{ Primary Site } & \multirow{2}{*}{$\begin{array}{l}\text { No. of } \\
\text { Patients }\end{array}$} & \multicolumn{3}{|c|}{ Response, No. (\%) } & \multirow{2}{*}{$\begin{array}{l}\text { ORR, \% } \\
(95 \% \mathrm{Cl})\end{array}$} \\
\hline & & CR & PR & $\mathrm{SD}>120$ Days & \\
\hline Colorectal & 37 & 0 & $14(38)$ & $4(11)$ & 38 (23 to 55 ) \\
\hline $\begin{array}{l}\text { Lung, } \\
\text { non-small- } \\
\text { cell }\end{array}$ & 16 & 0 & $2(13)$ & $2(13)$ & 13 (2 to 38 ) \\
\hline Bladder & 9 & $1(11)$ & $2(22)$ & $2(22)$ & 33 (8 to 70 ) \\
\hline Pancreas & 9 & 0 & $2(22)$ & $1(11)$ & 22 (3 to 60 ) \\
\hline Biliary & 7 & 0 & $2(29)$ & $3(38)$ & 29 (4 to 71$)$ \\
\hline Ovary & 8 & 0 & $1(13)$ & 0 & 13 (0 to 53 ) \\
\hline Uterus & 7 & 0 & 0 & 0 & 0 \\
\hline Salivary gland & 5 & 0 & $4(80)$ & 0 & $80(28$ to $>99)$ \\
\hline $\begin{array}{l}\text { Other }(11 \\
\text { sites }^{*}\end{array}$ & 16 & $1(6)$ & $1(6)$ & $3(19)$ & 13 (2 to 38$)$ \\
\hline Total & 114 & $2(2)$ & $28(25)$ & $16(14)$ & 26 (19 to 35$)$ \\
\hline
\end{tabular}

NOTE. $N=114$. Includes 12 patients with amplification/overexpression plus mutation.

Abbreviations: CR, complete response; ORR, objective response rate; PR, partial response; $\mathrm{SD}$, stable disease.

* Responses occurred in patients with adenocarcinomas of the prostate (one) and skin (apocrine; one). 

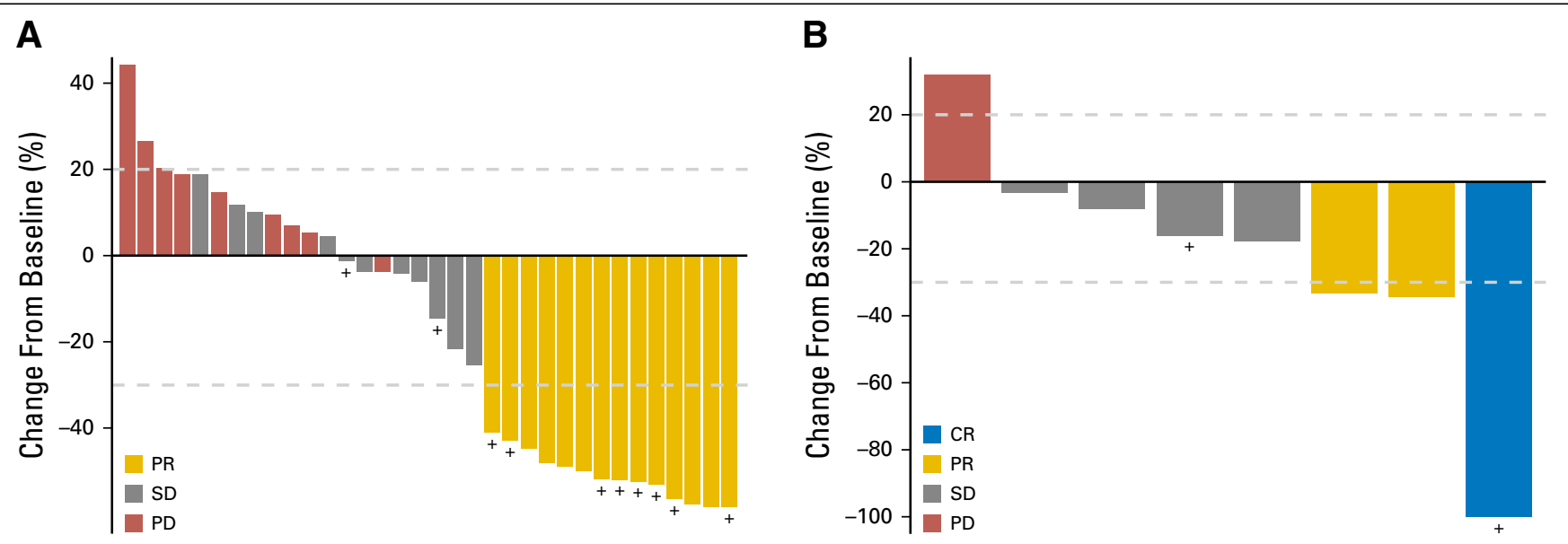

C

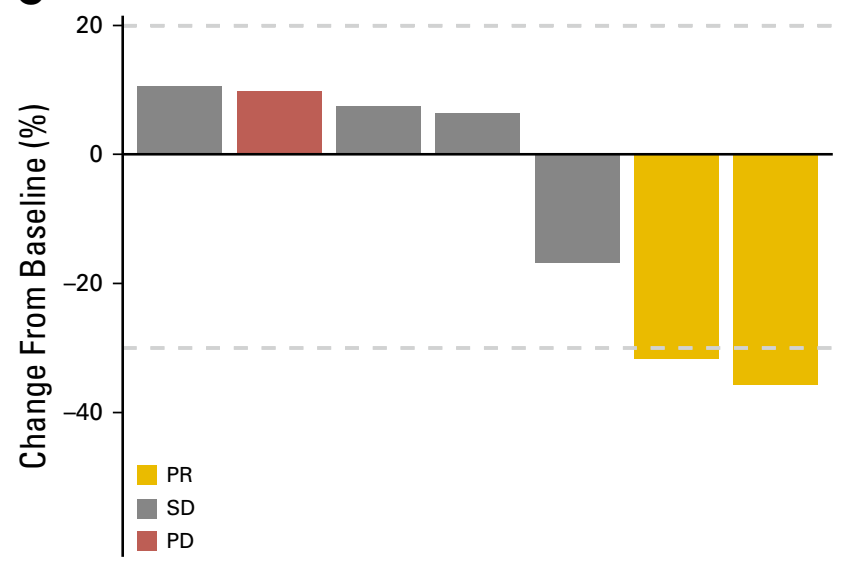

D

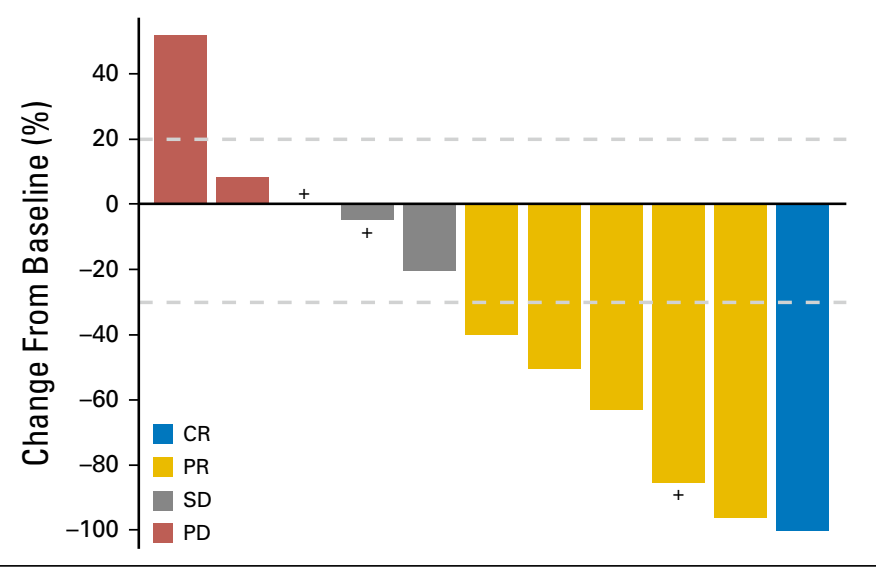

Fig 2. Waterfall plots of treatment response in patients with human epidermal growth factor receptor-2 (HER2)-amplified/overexpressing (A) colorectal, (B) bladder, and (C) biliary cancers, and (D) patients with murine sarcoma viral (V-raf) oncogene homolog B1 (BRAF) V600E-mutated non-small-cell lung cancer. Three patients with HER2-amplified/ overexpressing colorectal cancer, one with HER2-amplified/overexpressing bladder cancer, and three with BRAFV600E-mutated non-small-cell lung cancer were not evaluated for response and are not included. $\mathrm{CR}$, complete response; PD, progressive disease; PR, partial response; SD, stable disease. + indicates treatment is ongoing.

\section{Patients With HER2 Mutation}

Thirty-six patients received treatment with trastuzumab plus pertuzumab for tumors with HER2 mutations (without amplification/ overexpression); four of these patients (11\%; 95\% CI, 3\% to $26 \%$ ) had objective responses. Fourteen of the 36 patients with HER2-mutated tumors had NSCLC (adenocarcinoma, $\mathrm{n}=13$; adenosquamous, $\mathrm{n}=1$ ); in this group, three patients (21\%; 95\% CI, 5\% to 51\%) had PR and three had SD > 120 days. Only one of the other 22 patients with HER2mutated tumors responded to treatment with pertuzumab plus trastuzumab (biliary cancer).

\section{Patients With BRAF Mutations}

Results of treatment with vemurafenib differed markedly in tumors with BRAF V600 mutations when compared with those with other BRAF mutations. Of the 26 patients with BRAF V600 mutations (all V600E), objective responses were seen in 12 patients (46\%; 95\% CI, 27\% to 67\%) with six different tumor types (two CR, 10 PR; Table 4). In contrast, only one of 23 patients (4\%; 95\% CI, $0 \%$ to $22 \%$ ) with other non-V600 BRAF mutations had a PR (pancreas cancer with a CUX1-BRAF fusion). Nonresponding
BRAF mutations included: K601E $(\mathrm{n}=6), \mathrm{G} 464 \mathrm{~V}(\mathrm{n}=2), \mathrm{G} 469 \mathrm{~A}$ $(\mathrm{n}=2), \mathrm{G} 496 \mathrm{~A}(\mathrm{n}=2)$, N581S $(\mathrm{n}=2)$, and one each for G466V, G596R, G606E, L597Q, P731T, intron 9 rearrangement, intron 10 rearrangement, and MACF1- and WASFL-BRAF fusion.

Fourteen patients had refractory BRAF V600E-mutated NSCLC (adenocarcinoma, $\mathrm{n}=13$; sarcomatoid, $\mathrm{n}=1$ ), composing the largest tumor-pathway cohort in the BRAF group (Table 4). Six patients (43\%; 95\% CI, $18 \%$ to $71 \%$ ) had objective responses (one CR, five $\mathrm{PR}$ ), and two additional patients had SD $>120$ days (Fig 2D). The median DOR was 5 months (range, 4 to 14 months).

All other tumor-pathway cohorts with BRAF V600E mutations were small (four or fewer patients). Assessment of treatment efficacy in these groups is therefore not yet possible.

\section{Patients With Hedgehog Pathway or EGFR Mutations}

Twenty-one patients had mutations in the Hedgehog pathway (PTCH-1, $\mathrm{n}=18 ;$ SMO, $\mathrm{n}=3)$. Three patients had PRs to vismodegib (unknown primary cancer, $\mathrm{n}=1$; squamous skin cancer, $\mathrm{n}=1$; salivary gland cancer, $\mathrm{n}=1$ ); all three patients had PTCH-1-mutated tumors. Various EGFR mutations were present in the tumors of nine 


\begin{tabular}{|c|c|c|c|c|c|}
\hline \multirow[b]{2}{*}{ Primary Site } & \multirow{2}{*}{$\begin{array}{l}\text { No. of } \\
\text { Patients }\end{array}$} & \multicolumn{3}{|c|}{ Response, No. (\%) } & \multirow{2}{*}{$\begin{array}{l}\text { ORR, \% } \\
(95 \% \mathrm{Cl})\end{array}$} \\
\hline & & $\mathrm{CR}$ & PR & $\mathrm{SD}>120$ Days & \\
\hline $\begin{array}{l}\text { Lung, } \\
\text { non-small- } \\
\text { cell }\end{array}$ & 14 & $1(7)$ & $5(36)$ & $2(14)$ & 43 (18 to 71$)$ \\
\hline Ovary & 4 & 0 & $2(50)$ & $1(25)$ & 50 \\
\hline Colorectal & 2 & 0 & $1(50)$ & 0 & 50 \\
\hline $\begin{array}{l}\text { Unknown } \\
\text { primary }\end{array}$ & 1 & 0 & $1(100)$ & 0 & 100 \\
\hline $\begin{array}{l}\text { Thyroid } \\
\text { (anaplastic) }\end{array}$ & 1 & $1(100)$ & 0 & 0 & 100 \\
\hline $\begin{array}{l}\text { Head/neck } \\
\text { (larynx) }\end{array}$ & 1 & 0 & $1(100)$ & 0 & 100 \\
\hline Other (3 sites) & 3 & 0 & 0 & 0 & 0 \\
\hline Total & 26 & $2(8)$ & $10(38)$ & $3(12)$ & 46 (27 to 67$)$ \\
\hline
\end{tabular}

patients who received erlotinib; one of nine patients (with urethral adenocarcinoma) achieved a PR.

\section{DISCUSSION}

MyPathway was designed to evaluate the efficacy of targeted treatments in tumors harboring activating molecular alterations in the HER2, BRAF, EGFR, or Hedgehog pathways. In 230 patients who have been treated and evaluated, all four targeted treatments have produced meaningful responses, and 14 different tumor types outside of current US Food and Drug Administration (FDA) indications have responded. Completion of accrual to MyPathway is projected to take an additional 2 to 3 years. We believe that early publication of the encouraging results in several tumor-pathway cohorts will focus attention on these groups and accelerate definitive investigation. In addition, suggestions of activity in other small cohorts may guide the design of additional studies. As a whole, these early results provide further evidence of the feasibility and potential value of the basket study design in defining the optimal use of targeted therapies.

Patients with refractory, metastatic HER2-amplified/overexpressing colorectal cancer composed the largest tumor-treatment group in this study. The incidence of HER2 amplification/overexpression is $2 \%$ to $6 \%$ in advanced colorectal cancer, accounting for approximately 2,000 patients per year in the United States. ${ }^{25-27}$ In this study, the 37 patients with colorectal cancer treated with trastuzumab plus pertuzumab had an ORR of 38\% (95\% CI, 23\% to 55\%) and a median DOR of 11 months (95\% CI, 3 months to not estimable). The response rate and DOR in patients with refractory HER2amplified/overexpressing colorectal cancer indicate that HER2 is an important driver in this malignancy and compare favorably to the response rates of other drugs recently approved for use in refractory colorectal cancer. ${ }^{28-30}$ Dual HER2-targeted therapy was also effective in the HER2 Amplification for Colorectal Cancer Enhanced Stratification (HERACLES) trial, in which eight of 27 patients with HER2amplified/overexpressing, KRAS wild-type metastatic colon cancer $(30 \%)$ had objective responses to treatment with trastuzumab plus lapatinib. $^{26}$
Results are also encouraging in other HER2-amplified/ overexpressing tumor types, including bladder (33\% ORR), biliary tract (29\% ORR), and salivary gland ( $80 \%$ ORR). Although salivary duct carcinomas are rare, HER2 amplification/overexpression is common in these tumors. ${ }^{31,32}$ HER2 abnormalities in bladder and biliary cancers occur in $3 \%$ to $5 \%$ of patients. ${ }^{33,34}$ New treatment options are urgently needed in all of these treatment-refractory tumor types, and enlargement of these treatment groups is important.

Treatment with vemurafenib had notable activity in patients with refractory BRAF V600E-mutated cancers (46\% ORR). In $B R A F$ V600E-mutated NSCLC, treatment efficacy (43\% ORR; median DOR, 5 months) was similar to results previously reported in two other basket trials. ${ }^{15,35}$ In addition to the subsets defined by EGFR, ALK, and ROS-1 alterations, patients with BRAF V600Emutated NSCLC (approximately $2 \%$ of NSCLC $^{36}$ ) seem to be an important and targetable NSCLC subset. In contrast, the response rate for patients with non-V600E BRAF mutations was only $4 \%$, and accrual of these patients to MyPathway has been discontinued.

The role of basket trials in identifying patient populations for targeted therapy remains unclear. To date, most basket trials have reported mixed results, which may be due to the pathways being targeted, the methods of molecular testing, or the therapeutic agents being tested. The design of MyPathway maximizes the chance of success by using CLIA-approved molecular testing readily available in the clinic and by selecting FDA-approved targeted agents backed by more than a decade of translational science and clinical experience. Although not all genetic alterations are equally actionable, the four FDA-approved targeted therapies evaluated in MyPathway produced meaningful responses in patients with various tumor types involving well-defined driver alterations.

In conclusion, current results from MyPathway demonstrate potentially clinically meaningful activity for four approved targeted regimens in multiple tumor types harboring specific molecular alterations. Durable responses were seen in patients with colorectal, bladder, biliary, and salivary duct cancers with HER2 activation/ overexpression, in patients with NSCLC with BRAF V600E mutations, and in selected patients with $P T C H-1 / S M O$ or EGFR alterations. Treatment of additional patients in each of these groups is ongoing to better define the activity of these treatments and to further clarify the importance of molecular and histologic subgroups.

\section{AUTHORS' DISCLOSURES OF POTENTIAL CONFLICTS OF INTEREST}

Disclosures provided by the authors are available with this article at jco.org.

\section{AUTHOR CONTRIBUTIONS}

Conception and design: John D. Hainsworth, Mary Beattie

Provision of study materials or patients: John D. Hainsworth, David R. Spigel

Collection and assembly of data: Bongin Yoo

Data analysis and interpretation: All authors

Manuscript writing: All authors

Final approval of manuscript: All authors

Accountable for all aspects of the work: All authors 


\section{REFERENCES}

1. Slamon DJ, Leyland-Jones B, Shak S, et al: Use of chemotherapy plus a monoclonal antibody against HER2 for metastatic breast cancer that overexpresses HER2. N Engl J Med 344:783-792, 2001

2. Shepherd FA, Rodrigues Pereira J, Ciuleanu T, et al: Erlotinib in previously treated non-small-cell lung cancer. N Engl J Med 353:123-132, 2005

3. Chapman $\mathrm{PB}$, Hauschild $A$, Robert $\mathrm{C}$, et al: Improved survival with vemurafenib in melanoma with BRAF V600E mutation. N Engl J Med 364: 2507-2516, 2011

4. Sekulic A, Migden MR, Lewis K, et al: Pivotal ERIVANCE basal cell carcinoma (BCC) study: 12-month update of efficacy and safety of vismodegib in advanced BCC. J Am Acad Dermatol 72:1021-1026.e8, 2015

5. Cappuzzo F, Bemis L, Varella-Garcia M: HER2 mutation and response to trastuzumab therapy in non-small-cell lung cancer. N Engl J Med 354: 2619-2621, 2006

6. Forbes SA, Tang G, Bindal N, et al: COSMIC (the Catalogue of Somatic Mutations in Cancer): A resource to investigate acquired mutations in human cancer. Nucleic Acids Res 38:D652-D657, 2010 (suppl 1)

7. Masago K, Asato R, Fujita S, et al: Epidermal growth factor receptor gene mutations in papillary thyroid carcinoma. Int J Cancer 124:2744-2749, 2009

8. Masago $K$, Miura M, Toyama $Y$, et al: Good clinical response to erlotinib in a patient with anaplastic thyroid carcinoma harboring an epidermal growth factor somatic mutation, L858R, in exon 21. J Clin Oncol 29:e465-e467, 2011

9. Pakneshan $S$, Salajegheh $A$, Smith RA, et al: Clinicopathological relevance of BRAF mutations in human cancer. Pathology 45:346-356, 2013

10. Peters $S$, Michielin O, Zimmermann S: Dramatic response induced by vemurafenib in a BRAF V600E-mutated lung adenocarcinoma. J Clin Oncol 31:e341-e344, 2013

11. Wang $X D$, Inzunza $H$, Chang $H$, et al: Mutations in the hedgehog pathway genes SMO and PTCH1 in human gastric tumors. PLoS One 8: e54415, 2013
12. Xing $M$, Alzahrani AS, Carson $K A$, et al: Association between BRAF V600E mutation and mortality in patients with papillary thyroid cancer. JAMA 309:1493-1501, 2013

13. Conley BA, Doroshow JH: Molecular analysis for therapy choice: NCI MATCH. Semin Oncol 41: 297-299, 2014

14. Schilsky RL: Implementing personalized cancer care. Nat Rev Clin Oncol 11:432-438, 2014

15. Hyman DM, Puzanov I, Subbiah V, et al: Vemurafenib in multiple nonmelanoma cancers with BRAF V600 mutations. N Engl J Med 373:726-736, 2015

16. Hunter DJ, D'Agostino RB Sr: Let's not put all our eggs in one basket. N Engl J Med 373:691-693, 2015

17. Eisenhauer EA, Therasse $P$, Bogaerts J, et al: New response evaluation criteria in solid tumours: Revised RECIST guideline (version 1.1). Eur J Cancer 45:228-247, 2009

18. Yasuda H, Kobayashi S, Costa DB: EGFR exon 20 insertion mutations in non-small-cell lung cancer: preclinical data and clinical implications. Lancet Oncol 13:e23-e31, 2012

19. Andre F, Mardis E, Salm M, et al: Prioritizing targets for precision cancer medicine. Ann Oncol 25: 2295-2303, 2014

20. Perjeta (pertuzumab) [prescribing information] Genentech, South San Francisco, CA; 2016

21. Herceptin (trastuzumab) [prescribing information]. Genentech, South San Francisco, CA; 2016

22. Tarceva (erlotinib) [prescribing information]. Genentech, South San Francisco, CA; 2016

23. Zelboraf (vemurafenib) [prescribing information]. Genentech, South San Francisco, CA; 2016

24. Erivedge (vismodegib) [prescribing information] Genentech, South San Francisco, CA; 2015

25. Seo AN, Kwak Y, Kim DW, et al: HER2 status in colorectal cancer: Its clinical significance and the relationship between HER2 gene amplification and expression. PLoS One 9:e98528, 2014

26. Sartore-Bianchi $A$, Trusolino $L$, Martino $C$, et al: Dual-targeted therapy with trastuzumab and lapatinib in treatment-refractory, KRAS codon 12/13 wild-type, HER2-positive metastatic colorectal cancer (HERACLES): A proof-of-concept, multicentre, open-label, phase 2 trial. Lancet Oncol 17:738-746, 2016
27. Richman SD, Southward $K$, Chambers $P$, et al: HER2 overexpression and amplification as a potential therapeutic target in colorectal cancer: Analysis of 3256 patients enrolled in the QUASAR, FOCUS and PICCOLO colorectal cancer trials. J Pathol 238: 562-570, 2016

28. Grothey A, Van Cutsem E, Sobrero A, et al: Regorafenib monotherapy for previously treated metastatic colorectal cancer (CORRECT): An international, multicentre, randomised, placebo-controlled, phase 3 trial. Lancet 381:303-312, 2013

29. Mayer RJ, Van Cutsem E, Falcone $A$, et al: Randomized trial of TAS-102 for refractory metastatic colorectal cancer. N Engl J Med 372:1909-1919, 2015

30. Van Cutsem E, Peeters M, Siena S, et al: Openlabel phase III trial of panitumumab plus best supportive care compared with best supportive care alone in patients with chemotherapy-refractory metastatic colorectal cancer. J Clin Oncol 25:1658-1664, 2007

31. Glisson B, Colevas AD, Haddad R, et al: HER2 expression in salivary gland carcinomas: Dependence on histological subtype. Clin Cancer Res 10:944-946, 2004

32. Cornolti G, Ungari $M$, Morassi $M L$, et al: Amplification and overexpression of HER2/neu gene and HER2/neu protein in salivary duct carcinoma of the parotid gland. Arch Otolaryngol Head Neck Surg 133:1031-1036, 2007

33. Laé $M$, Couturier J, Oudard $S$, et al: Assessing HER2 gene amplification as a potential target for therapy in invasive urothelial bladder cancer with a standardized methodology: Results in 1005 patients. Ann Oncol 21:815-819, 2010

34. Harder J, Waiz O, Otto F, et al: EGFR and HER2 expression in advanced biliary tract cancer. World J Gastroenterol 15:4511-4517, 2009

35. Blay J-Y, Mazieres J, Perol D, et al: Vemurafenib in non-melanoma V600 and non-V600 BRAF mutated cancers: First results of the ACSE trial. Ann Oncol 27:55PD, 2016 (suppl 6)

36. Marchetti $A$, Felicioni $L$, Malatesta $S$, et al: Clinical features and outcome of patients with nonsmall-cell lung cancer harboring BRAF mutations. J Clin Oncol 29:3574-3579, 2011

\section{Affiliations}

John D. Hainsworth, David R. Spigel, and Howard Burris, Sarah Cannon Research Institute; Tennessee Oncology, Nashville, TN; Funda Meric-Bernstam, University of Texas MD Anderson Cancer Center, Houston, TX; Charles Swanton, Francis Crick Institute, London, United Kingdom; Herbert Hurwitz, Duke University Medical Center, Durham, NC; Christopher Sweeney, Dana-Farber Cancer Institute, Harvard Medical School, Boston, MA; Ron Bose, Washington University School of Medicine, St Louis, MO; Bongin Yoo, Alisha Stein, and Mary Beattie, Genentech, South San Francisco; and Razelle Kurzrock, Moores Cancer Center, University of California San Diego, San Diego, CA.

The MyPathway study was supported by Roche/Genentech.

\section{Support}

\section{Prior Presentation}

Presented in part at the American Society of Clinical Oncology Gastrointestinal Cancers Symposium 2016, San Francisco, CA, January 21-23, 2016; American Society of Clinical Oncology 52nd Annual Meeting, Chicago, IL, June 3-7, 2016; American Society of Clinical Oncology Gastrointestinal Cancers Symposium 2017, San Francisco, CA, January 19-21, 2017; American Society of Clinical Oncology Genitourinary Cancers Symposium 2017, Orlando, FL, February 16-18, 2017; and American Society of Clinical Oncology 53rd Annual Meeting, Chicago, IL, June 2-7, 2017. 


\section{AUTHORS' DISCLOSURES OF POTENTIAL CONFLICTS OF INTEREST}

Targeted Therapy for Advanced Solid Tumors on the Basis of Molecular Profiles: Results From MyPathway, an Open-Label, Phase IIa Multiple Basket Study

The following represents disclosure information provided by authors of this manuscript. All relationships are considered compensated. Relationships are self-held unless noted. I = Immediate Family Member, Inst = My Institution. Relationships may not relate to the subject matter of this manuscript. For more information about ASCO's conflict of interest policy, please refer to www.asco.org/rwc or ascopubs.org/jco/site/ifc.

John D. Hainsworth

Research Funding: Genentech, Novartis, Eli Lilly, AstraZeneca, Astellas Pharma, Johnson \& Johnson, Celgene

\section{Funda Meric-Bernstam}

Consulting or Advisory Role: Genentech, Inflection Biosciences, Pieris Pharmaceuticals, Clearlight Diagnostics, Darwin Health

Research Funding: Novartis, AstraZeneca, Taiho Pharmaceutical, Genentech, Calithera Biosciences, Debiopharm, Bayer AG, Aileron Therapeutics, PUMA Biotechnology, CytomX Therapeutics, Jounce Therapeutics, Zymeworks, Effective Pharmaceuticals, Curis

\section{Charles Swanton}

Stock or Other Ownership: Epic Sciences, Apogen Biotechnologies, GRAIL, Achilles Therapeutics

Honoraria: Roche, Boehringer Ingelheim, GlaxoSmithKline, Eli Lilly, Celgene, Ono Pharmaceutical, SERVIER, Pfizer

Consulting or Advisory Role: Genentech

Research Funding: Boehringer Ingelheim

Patents, Royalties, Other Intellectual Property: Patent WO2016174085

A1: Method for treating cancer based on identification of clonal neoantigens; Founder of Achilles Therapeutics: a biotechnology company funded by Syncona/Wellcome Trust to target clonal neoantigens through vaccine and cell therapy approaches; Patent WO2017042394 A1: Methods for identifying a patient with cancer who is suitable for treatment with an immune checkpoint intervention

\section{Herbert Hurwitz}

Consulting or Advisory Role: Genentech, Bristol-Myers Squibb, Eli Lilly, Novartis, Incyte, TRACON Pharmaceuticals, Acceleron Pharma, GlaxoSmithKline, OncoMed Pharmaceuticals

Research Funding: Genentech, GlaxoSmithKline, Novartis, TRACON Pharmaceuticals, Bristol-Myers Squibb, Regeneron Pharmaceuticals, Eli Lilly, Macrogenics, National Cancer Institute

\section{David R. Spigel}

Consulting or Advisory Role: Genentech (Inst), Celgene (Inst), Novartis (Inst), Eli Lilly (Inst), Pfizer (Inst), Bristol-Myers Squibb (Inst), AstraZeneca (Inst)

Research Funding: Genentech (Inst), Pfizer (Inst), Novartis (Inst), BristolMyers Squibb (Inst), Eli Lilly (Inst), Celgene (Inst)

Travel, Accommodations, Expenses: Bristol-Myers Squibb, Peregrine Pharmaceuticals

\section{Christopher Sweeney}

Stock or Other Ownership: Leuchemix

Consulting or Advisory Role: Sanofi, Janssen Biotech, Astellas Pharma, Bayer, Genentech, AstraZeneca, Pfizer

Research Funding: Janssen Biotech (Inst), Astellas Pharma (Inst), Sanofi (Inst), Bayer (Inst), Sotio (Inst)

Patents, Royalties, Other Intellectual Property: Leuchemix, Parthenolide, Dimethylaminoparthenolide, Exelixis: Abiraterone plus cabozantinib combination

\section{Howard Burris}

Research Funding: Genentech (Inst), Bristol-Myers Squibb (Inst), Incyte (Inst), Tarveda Therapeutics (Inst), Mersana Therapeutics (Inst), AstraZeneca (Inst), MedImmune (Inst), Macrogenics (Inst), Novartis (Inst), Boehringer Ingelheim (Inst), Eli Lilly (Inst), Seattle Genetics (Inst), AbbVie (Inst), Bayer (Inst), Celldex Therapeutics (Inst), Merck (Inst), Celgene (Inst), Agios Pharmaceuticals (Inst), Jounce Therapeutics (Inst)

\section{Ron Bose}

Honoraria: Genentech, Novartis

Consulting or Advisory Role: Genentech

Bongin Yoo

Employment: Genentech

Stock or Other Ownership: Roche

Alisha Stein

Employment: Genentech

Stock or Other Ownership: Roche

Mary Beattie

Employment: Genentech

Stock or Other Ownership: Genentech

Travel, Accommodations, Expenses: Genentech

\section{Razelle Kurzrock}

Stock or Other Ownership: Actuate Therapeutics, CureMatch

Honoraria: Cedars-Sinai, National Comprehensive Cancer Network, American Association for Cancer Research, Yale Cancer Center, XBiotech, Pancreatic Cancer Action Network, Sylvester Cancer Center, Mayo Clinic Cancer Center, Kaiser Permanente, Health Advances, Wiley, Scripps Translational Research Institute, Defined Health, CME Education Resources, Avera Health, Genentech, LOXO Oncology, Health Advances Consulting or Advisory Role: Sequenom, Actuate Therapeutics, XBiotech, Genentech, LOXO Oncology

Research Funding: EMD Serono (Inst), Centocor Ortho Biotech (Inst), GlaxoSmithKline (Inst), Exelixis (Inst), XBiotech, Novartis (Inst), Merck (Inst), Genentech (Inst), Genentech Mypath (Inst), Foundation Medicine (Inst), Pfizer (Inst), Guardant Health (Inst), Sequenom (Inst), Merck Serono (Inst), Incyte

Patents, Royalties, Other Intellectual Property: Patent US7968115 B2: Liposomal curcumin for treatment of cancer; Patent US8784881 B2: Liposomal curcumin for treatment of diseases

Travel, Accommodations, Expenses: Worldwide Innovative Networking (WIN) in Personalized Cancer Medicine, EMD Serono, Gateway Research Advisory Committee, AACR, Quintiles, Association of American Cancer Institutes, Guardant Health, Global Source Ventures/Novena Therapeutics, American Society for Clinical Pharmacology and Therapeutics, Meyers Consulting, Food and Drug Administration - Orange County Regulatory Affairs, Genentech, Orbimed/Global Source Ventures, Sylvester Cancer Center, Journal of Precision Medicine, CureMatch, Lynx Group, Mayo Clinic Cancer Center, Kaiser Permanente, Pancreatic Cancer Action Network, Cedars-Sinai, MedImmune/JK Associates Medical Communications Group, Federation of the Israel Societies for Experimental Biology, Defined Health 


\section{Acknowledgment}

We thank the patients, families, clinicians, and study teams who have participated in the MyPathway study. We also thank Melissa Brammer for her contributions to the study and Coen Bernaards, David Moorman, and Bryan Lange for their assistance with the biostatistical analysis. MyPathway was funded by Roche/Genentech. Support for third-party writing assistance was provided by Tiffany DeSimone, of CodonMedical, an Ashfield Company, part of UDG Healthcare, and was funded by Roche/Genentech. 